\title{
Oligonucleotide-functionalized hydrogels as stimuli responsive materials and biosensors
}

\author{
Juewen Liu* \\ Department of Chemistry, Waterloo Institute for Nanotechnology, University of Waterloo, Waterloo, \\ Ontario, N2L 3G1, Canada \\ Email: liujw@uwaterloo.ca
}

\begin{abstract}
Hydrogels are crosslinked hydrophilic polymers that undergo swelling in water. The gel volume is affected by many environmental parameters including temperature, $\mathrm{pH}$, ionic strength, and solvent composition. Therefore, these factors have been traditionally used for making smart hydrogels. DNA, on the other hand, is a special block copolymer. Incorporation of DNA within a hydrogel network can have several important effects. For example, DNA can serve as a reversible crosslinker modulating the mechanical and rheological properties of a hydrogel. Second, DNA can selectively bind to a variety of different molecules. Attaching these binding DNAs inside (aptamers) the hydrogel makes it possible to expand the range of stimuli to chemical and biological molecules. At the same time, the gel matrix can also improve DNA-based sensors and materials. For example, the hydrogel can be dried for storage and rehydrated prior to use and the immobilized DNAs are protected from nuclease cleavage. The gel backbone property can also be tuned to affect the interaction between DNA and other molecules. The rational functionalization of DNA in hydrogels has generated a diverse range of smart materials. In the last 15 years, the field has made tremendous progress and some of the recent developments have been summarized in this review. Challenges and possible future directions are also discussed.
\end{abstract}


Juewen Liu is an Assistant Professor at the Department of Chemistry, University of Waterloo. Dr. Liu received his $\mathrm{PhD}$ in Chemistry from the University of Illinois at Urbana-Champaign in 2005. He is interested in studying DNA functionalized soft materials and nanoparticles for analytical and biomedical applications.

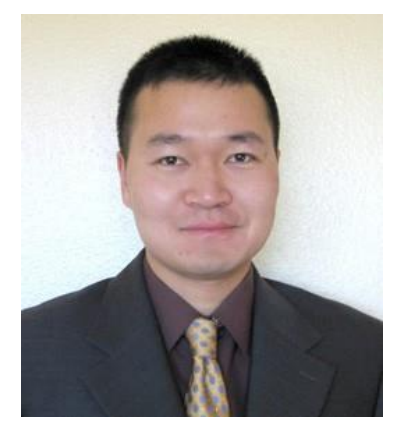

\section{Juewen Liu}

\section{Introduction}

Hydrogels are crosslinked hydrophilic polymer networks that possess many attractive physical properties. $^{1-9}$ A hydrogel can absorb and retain a large amount of water, up to several hundred fold of the gel dry mass. The amount of retained water or swelling is reflected by the gel volume and is influenced by solution conditions, such as temperature, $\mathrm{pH}$, ionic strength, solvent composition, as well as light and electric field. ${ }^{10}$ Therefore, a number of smart hydrogels have been prepared to be responsive to these stimuli. For example, hydrogels polymerized from the monomer Nisopropylacrylamide (NIPAm) show a large temperature-dependent volume change. ${ }^{11}$ If the temperature is below the lower critical solution temperature (LCST) for pNIPAM, the water-polymer interaction is strong to swell the gel. Above this temperature, the hydrogen bonds between the gel and water break and the polymer interacts with itself through the hydrophobic interaction of the isopropyl group to decrease the gel volume. ${ }^{12}$ In another example, a polyacrylic acid gel swells more at higher $\mathrm{pH}$, where the polymer chains are highly negatively charged. If the ionic strength is increased, the gel swelling is reduced due to the screening of the repulsive charge. These stimuli responsive properties have been widely used to make stimuli responsive materials 
for various applications including controlled drug release systems, sensors, cell culture substrates, flow control, and other smart materials. ${ }^{13-17}$

With significant progress being made in the design of responsive hydrogels, however, the choice of input stimuli has been quite limited. For many bioanalytical and biomedical applications, it is highly desirable to have hydrogels responsive to small molecule metabolites, nucleic acids, proteins, and other chemical and biological inputs. For example, insulin release in response to glucose concentration change is needed for treating diabetes. Towards this goal, various biomolecular interactions have been employed. ${ }^{18-21}$ Majority of the published work has focused on peptide and protein-functionalized hydrogels. In the seminal work by Miyata et al, IgG and its antibody were covalently attached to polymer chains, which subsequently formed a hydrogel with the binding between these two molecules. ${ }^{18}$ Addition of free $\operatorname{IgG}$ induced gel swelling because free $\operatorname{IgG}$ competed with the immobilized IgG to reduce the crosslinking density.

While proteins have been the major player in realizing biological functions in terms of catalysis and molecular recognition, our understanding of DNA has drastically expanded in the past twenty years from a pure genetic material to a versatile functional polymer. ${ }^{22-28}$ Thanks to the invention of solid phase DNA synthesis, DNA with arbitrary sequences up to 100 nucleotides containing various chemical modifications can be routinely synthesized. This has fueled the development of DNA nanotechnology leading to the demonstration of remarkable DNA structures and devices. ${ }^{22,29,30}$ DNA is also used as a template to assemble various inorganic nanoparticles to form nanoparticle oligomers, ${ }^{25}$ large aggregates, ${ }^{26}$ crystals, ${ }^{31,32}$ and various $2-\mathrm{D}$ periodic structures. ${ }^{23,33}$ At the same time, the invention of polymerase chain reaction (PCR) has revolutionized nucleic acid biotechnology. Combinatorial selection methods have been developed to isolate DNAs with catalytic and molecular recognition functions. ${ }^{34-36}$ Therefore, chemical and biological functions that used to be exclusive to proteins can now be realized using DNA. 
Compared to proteins, DNA has the following advantages that make it particularly attractive in making stimuli responsive materials. First, DNA is a very stable molecule. Even though DNA can be denatured by heating, re-naturation readily occurs at a lower temperature. In contrast, antibodies and protein enzymes are usually irreversibly denatured upon heating. Second, DNA folding obeys simple base pairing rules and DNA secondary structures can be readily predicted. Materials can be designed with minimal information about the 3-D structure of DNA. Third, the ease of DNA synthesis and modification allows the incorporation of various optical and electrochemical signaling mechanisms; while site specific labeling of proteins is quite challenging. With the development in both DNA materials science and DNA biotechnology, many DNA-based stimuli-responsive materials have been prepared, ${ }^{27,37-40}$ including hydrogels. DNA-functionalized hydrogel is still a relatively new field where the first publication appeared in $1996,{ }^{41}$ and the second one appeared six years later. ${ }^{42}$ In the last several years, however, a lot of exciting new developments have been reported showing applications in controlled protein release, sensing, and in making smart materials.

Since hydrogels are prepared in solution, they can be readily made into many different physical forms including monoliths, thin films, and micro/nanoparticles. Each form has a different cargo loading capacity, release kinetics and mechanical property. For example, micro/nanoparticles are useful for drug delivery while thin films are ideal for cell culturing and for making various fast response transducers. Monoliths, on the other hand, are easy to handle, to observe, and to incorporate into devices. As a result, most of the reported DNA-functionalized hydrogels are monolithic materials, which will also be the main focus of this review. The involved design principles, however, are also applicable to other gel forms. This review is also focused on oligonucleotide-functionalized hydrogels where the DNA sequence is usually less than 100 nucleotides. Genomic DNAs have also been used to form hydrogels with cationic surfactants, ${ }^{43-48}$ polymers, ${ }^{49-51}$ chemical crosslinkers, ${ }^{52,53}$ and inorganic materials. ${ }^{54}$ These gels are based on non-specific electrostatic or hydrophobic interactions and are not reviewed here. In addition, hydrogels containing DNA for gene delivery purposes are also excluded from this review. ${ }^{55-57}$ DNA-functionalized 
hydrogels can respond to stimuli in several different ways. We start with the sol-gel transition, followed by the reversible gel volume change, and end with gels that can generate optical signals.

\section{DNA as a functional polymer}

Apart from its biological function, DNA is a block copolymer with just four kinds of monomers (A, T, C, G) linked by the phosphodiester bond. Each phosphate carries a negative charge and DNA is a polyanion. In an aqueous solution, the B-form double-stranded (ds) DNA has a diameter of $\sim 2 \mathrm{~nm}$ and a persistent length of $\sim 50 \mathrm{~nm}$, allowing it to be treated as a rigid rod. By heating ds-DNA above its melting temperature, two flexible single-stranded (ss) DNAs are generated. This basic understanding has allowed DNA to be widely used as a special polymer that can provide predictable binding and can be controlled with sub-nanometer precision.

Unlike most synthetic polymers, the arrangement of the four monomer units in DNA can be preciously controlled, which directly affects the DNA property. For example, certain ss-DNA can

selectively bind to molecular targets and these binding DNAs are known as aptamers. ${ }^{34,35}$ Figure 1A shows the secondary structure of a thrombin binding aptamer that folds into a G-quadruplex. Thrombin is an important serine protease and is often used as a model protein to test aptamer binding. Figures 1B and $\mathrm{C}$ present the structures of adenosine and cocaine aptamers, respectively; both have a dissociation constant $\left(K_{d}\right)$ in the low $\mu \mathrm{M}$ range. ${ }^{58,59}$ Figure $1 \mathrm{D}$ shows a thymine rich DNA capable of binding $\mathrm{Hg}^{2+}$ to form $\mathrm{T}-\mathrm{Hg}^{2+}-\mathrm{T}$ base pairs. ${ }^{60,61}$ Aptamers can rival antibodies in terms of binding affinity, specificity, and range of target molecules. ${ }^{62,63}$ In addition to the four examples shown here, hundreds of aptamers have been isolated to bind to many different target molecules. ${ }^{62,63}$ 


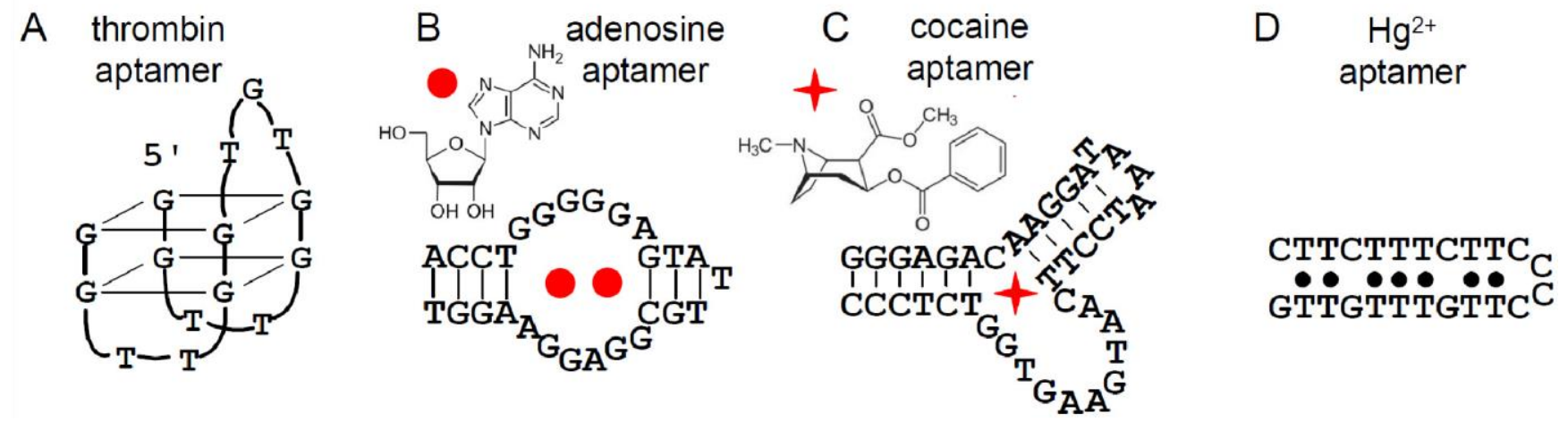

Figure 1. Examples of DNA aptamers for thrombin (A), adenosine or ATP (B), cocaine (C) and mercury ions (D). In (B), each aptamer binds two adenosine molecules. In (D) there are seven $\mathrm{Hg}^{2+}$ binding sites in each DNA indicated by the dots.

\section{Conjugate chemistry}

DNA can be modified with many functional groups including amino, biotin, acrydite, azide, and thiol to accommodate a variety of attachment chemistry. Most of DNA-functionalized gels are acrylic-based. To date, two methods have been used to covalently incorporate DNA into a hydrogel. In the first case, an amino-modified DNA reacts with a monomer or a polymer containing a reactive succinimidyl ester to form an amide bond (Figure 2A). As a result, the DNA is modified with an acrydite group or incorporated into the gel. This reaction can take place before or after hydrogel/polymer formation, which may allow a uniform DNA distribution within the gel or selectively attaching DNA only to the gel surface. In the second approach, acrydite-modified DNA can be directly incorporated during gel formation as shown in Figure 2B. An acrydite DNA has a similar activity or reactivity as free acrylamide monomers allowing high incorporation efficiency. ${ }^{64}$ Unlike the amino-modified DNA, where both $3^{\prime}$ and $5^{\prime}$-modifications are available, the acrydite modification is currently only available on the 5 -end.

In addition to forming covalent linkages, DNAs can also be entrapped in the hydrogel network. The advantage of entrapment is the reduced cost associated with modified DNA synthesis. However, entrapped DNA can still leach out via diffusion. Being a polyanion, the diffusion of entrapped DNA may 
be reduced by increasing the DNA length, gel percentage, or by using cationic monomers to form the gel. ${ }^{65}$ The diffusion may be further reduced by linking DNA to large proteins and microparticles. ${ }^{66}$

A

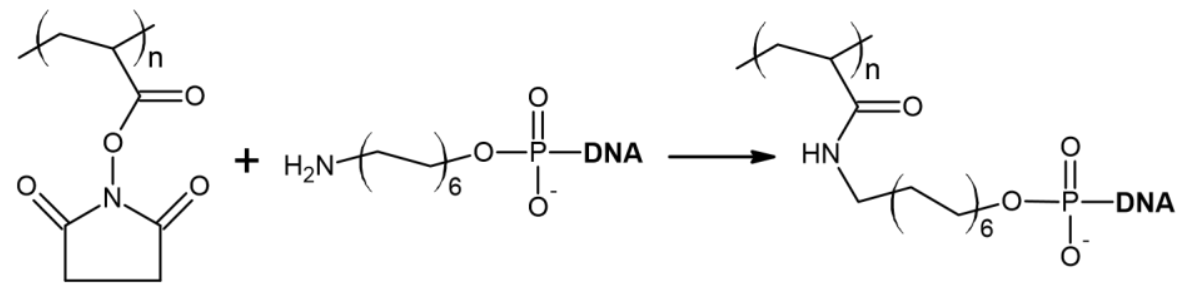

B

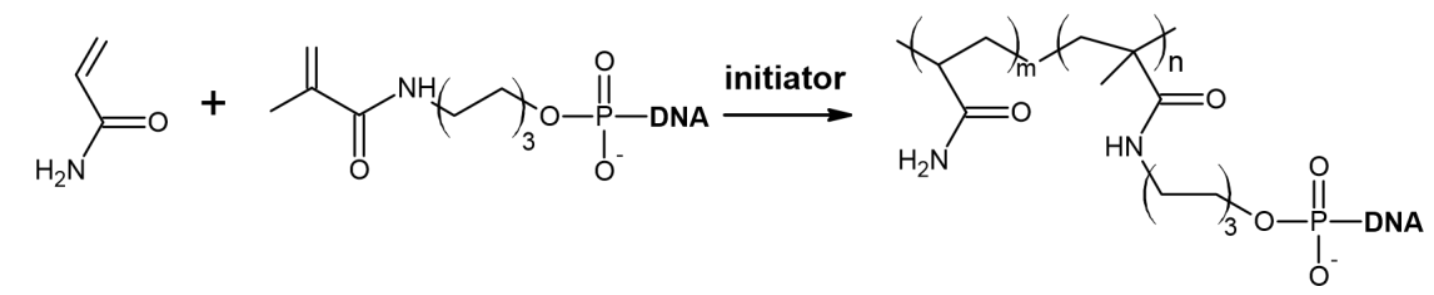

Figure 2. The two types of conjugate chemistry for covalent attaching DNA to hydrogel. (A) Aminomodified DNA reacts with a polymer containing succinimidyl ester on the backbone. (B) Copolymerization of acrydite-modified DNA into polyacrylamide.

\section{Stimuli-responsive sol/gel transition}

In the sol-gel process, gels are formed from solution and can be made into different sizes and shapes. Physical properties such as viscosity drastically change upon gel formation and can be easily observed. Typically, chemical crosslinkers such as $N, N^{\prime}$-methylene-bis-acrylamide are used to form acrylamidebased gels and such gels cannot be easily degraded or cleaved to return to the sol state, resulting in a permanent 3-D polymer network. Instead of using bis-acrylamide, if DNA hybridization is employed to crosslink polymer chains, reversible and thermally responsive gels can be prepared. The first work in this field was reported by Nagahara and Matsuda in 1996. ${ }^{41}$ They prepared a block copolymer using $N, N$-dimethylacrylamide and $N$-acrylaoyloxysuccinimide. The latter monomer can react with aminomodified DNAs (see Figure 2A). Two polymer chains were prepared using different DNA sequences. A hydrogel was formed after the addition of a linker DNA to assemble the two DNA strands as shown in Figure 3A. The gel reversibly transitioned into sol upon heating because of DNA melting. 
In principle, this system can be used to detect the linker DNA. Practically, however, this does not appear to be a very attractive method for DNA detection since the amount of linker DNA required to induce the sol-gel transition is at $\mathrm{mM}$ level. Nevertheless, this pioneer work has demonstrated the feasibility of forming hydrogels with DNA and has worked out the conjugate chemistry. Interestingly, in the same year, DNA was also linked to gold nanoparticles (AuNPs) and the assembly of nanoparticle "gels" with a linker DNA was demonstrated. ${ }^{25,26}$ Apparently, the AuNP work has received far more attention due to the attractive optical property of AuNPs that makes ultrasensitive colorimetric DNA detection possible.27, 67-69

Six years later in 2002, a conference proceeding was published by the Langrana group, ${ }^{42}$ followed by a full paper in 2004 reporting the mechanical property of such DNA-crosslinked hydrogels. ${ }^{70,71}$ They $^{7}$ employed a system shown in Figure 3B, where the linker DNA contained an overhang (in red). This overhang can facilitate the removal of the linker in the presence of the complementary DNA (c-DNA). This reaction occurs spontaneously at constant temperature, omitting the need for heating. The authors measured the viscosity of the system as a function of both crosslinking density and temperature. They found that gels formed only if the crosslink concentration was higher than $\sim 23 \%$. The viscosity of the system also decreased with increasing temperature. Addition of the c-DNA to the gel induced a faster leakage of entrapped fluorophores. This work clearly demonstrated the ability of DNA crosslinked gels to be responsive to multiple stimuli, not only temperature but also the c-DNA. Later, Simmel and coworkers used fluorescent quantum dots (QDs) as probes and the diffusion of QDs within the gel was monitored by fluorescence microscopy and fluorescence correlation spectroscopy. ${ }^{72}$ The kinetics of the gel-to-sol transition induced by the c-DNA was quite slow (likely due to the relatively large size of DNA) and several hours were needed to observe improved diffusion kinetics of the entrapped QDs. Towards 
the end of the paper, the authors mentioned that introducing aptamers or aptazymes to such hydrogels may be more practical for controlled drug release applications.

The use of aptamers to prepare stimuli-responsive inorganic materials has been reported using AuNPs, ${ }^{73-76} \mathrm{QDs}^{77}$ and magnetic nanoparticles. ${ }^{78}$ Tan and co-workers first showed that a gel-to-sol transition can be achieved using an aptamer-assembled hydrogel. ${ }^{79}$ In Figure $3 \mathrm{C}$, the green strand represents the aptamer for adenosine. In the absence of adenosine, the aptamer DNA behaved similarly to a normal DNA participating in crosslinking the hydrogel. Addition of adenosine induced aptamer folding to break the crosslink. Since adenosine is a small molecule, its diffusion into the monolithic gel was much faster compared to the c-DNA as shown in Figure 3B. If AuNPs were entrapped inside the gel as a color indicator, $2 \mathrm{mM}$ adenosine completely dissolved the gel within $15 \mathrm{~min}$ (Figure 3D, E). The authors also demonstrated the use of the thrombin binding aptamer (Figure 1A) to form hydrogel and thrombin induced gel-to-sol transition with a slower gel dissolution rate because of the larger size of thrombin.

Gel

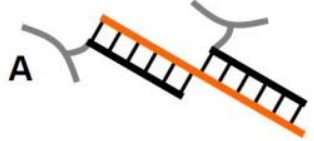

Sol
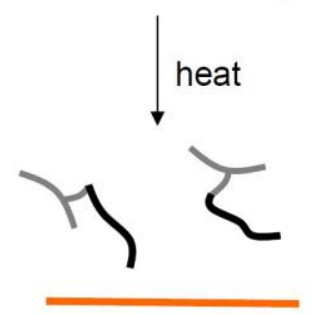

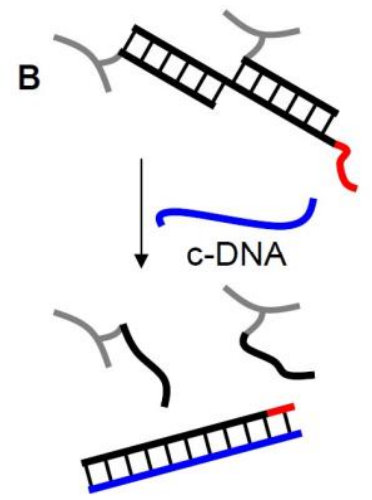

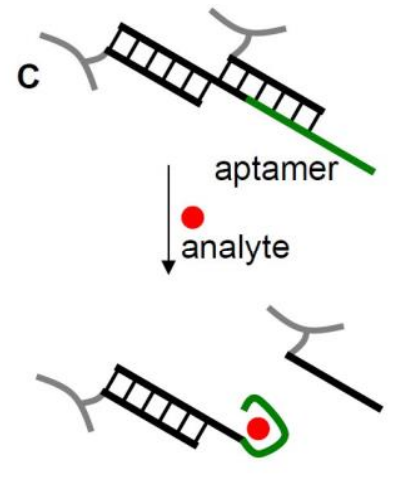

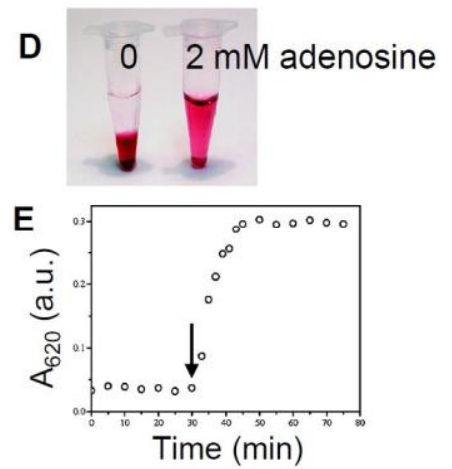

Figure 3. Stimuli responsive gel-to-sol transition in DNA-functionalized hydrogels. (A) Transition induced by change in temperature. (B) Transition induced by addition of a c-DNA. (C) Transition induced by a small molecule where the linker DNA contained the aptamer sequence. (D) A photograph of the adenosine responsive hydrogel with entrapped gold nanoparticles (red color) in the presence or absence of adenosine. (E) Addition of adenosine induced the gel-to-sol transition in $~ 15 \mathrm{~min}$. 
While AuNPs can serve as a visual indicator for gel dissolution as shown in Figure 3D, a high concentration of AuNPs can give an intense background color, which makes the detection of a small amount of released AuNPs difficult. As a result, this detection method is not analytically sensitive. To solve this problem, the Yang and Tan groups entrapped an enzyme amylase within a cocaine aptamer crosslinked hydrogel (Figure 4) ${ }^{80}$ In the presence of cocaine, amylase was released due to hydrogel dissolution to convert amylose into small sugar molecules, which were colorless in the presence iodine. Otherwise an intense blue color was observed due to the well-known amylose/iodine interaction. Since each enzyme can catalyze the conversion of many amylose molecules, the gel does not have to completely dissolve to show this effect. As a result, even $2 \mu \mathrm{M}$ cocaine can be visually detected. Recently, Zhang, Tan, and co-workers also engineered a photoresponsive hydrogel crosslinked by DNA ${ }^{81}$ The linker DNA contained azobenzenes, which can stabilize the DNA duplex in the trans-form but destabilize it in the cisform. ${ }^{82}$

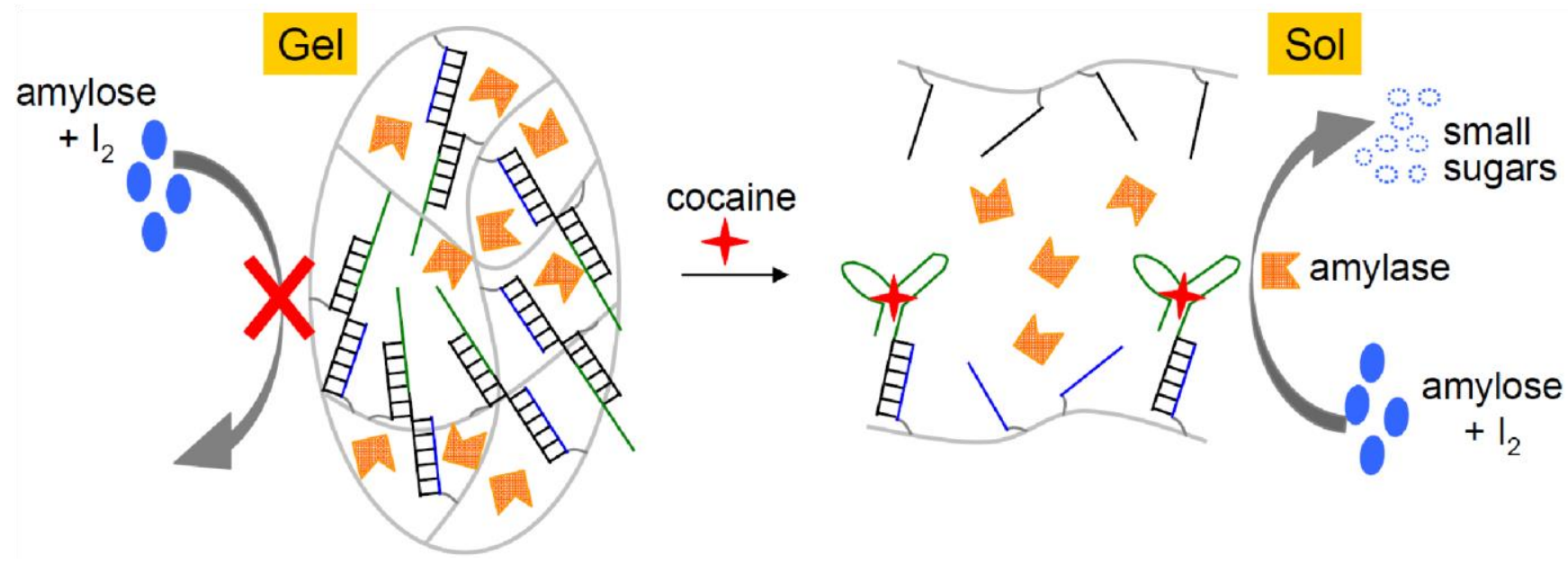

Figure 4. Detection of cocaine using a hydrogel containing entrapped amylase. In the gel state, the enzyme cannot digest amylose and a blue color is observed in the presence of iodine. In the presence of cocaine, the gel network is disrupted to release amylose to give a transparent solution. 
In addition to making sensors, DNA crosslinked hydrogels have also been used for controlled release. For example, Mi and co-workers prepared a DNA-functionalized polyacrylamide hydrogel, where the thrombin aptamer was included in the DNA for crosslinking the gel. Therefore, this gel was able to retain $>90 \%$ of the thrombin protein even after soaking in buffer for a week. ${ }^{83}$ Addition of the cDNA inactivated the aptamer and induced the gel-to-sol transition, resulting in complete release of thrombin. The same group further demonstrated that it is possible to selectively retain thrombin in the gel while releasing bovine serum albumin using this system. ${ }^{84}$ Therefore, they were able to isolate thrombin from a mixture of these two proteins. By attaching an aptamer for platelet-derived growth factor (PDGF) to a hydrogel backbone, Wang and co-workers found that the gel released PDGF at a slower rate compared to the non-functionalized gels. Several different aptamers with different PDGF binding affinities were tested and the release rate was found to be proportional to the aptamer $K_{d}$ 's. ${ }^{85}$ Based on this understanding, the Wang group further demonstrated a pulsatile protein release system where protein release was triggered by the addition of the c-DNA. ${ }^{66}$ In addition to proteins, controlled release of DNA was demonstrated by Venkatesh et al. ${ }^{86}$ In this work, an acrydite-modified DNA was attached to the hydrogel backbone and the DNA to be released was hybridized to the acrydite DNA. Release was achieved via restriction enzyme cleavage. The released fragment can perform various biochemical functions, depending on the DNA sequence.

The gels reviewed so far contained both synthetic polymers and DNA, where the gel backbone is made-up mainly by synthetic polymers. The role of DNA ranges from crosslinkers to functional side chains. Interestingly, it is also possible to prepare hydrogels just with DNA. ${ }^{87,88}$ Starting with 3 -way or 4-way branched DNA structures with sticky ends as monomers, the Luo group successfully prepared hydrogels in the presence of a DNA ligase. ${ }^{88}$ This system was tested as a controlled drug release system. The same group also entrapped an in-vitro transcription and translation system, which showed a 300fold 
higher efficiency compared to the same reaction in solution. ${ }^{89}$ Instead of using the DNA ligase to form covalent linkages between DNA building blocks, the i-motif DNA formation was harnessed by Liu and co-workers to make DNA gels. As shown in Figure 5A, the three-way DNA junction monomer contained C-rich overhangs. ${ }^{90}$ At low $\mathrm{pH}$, these $\mathrm{C}$-rich ends self-assemble to form the i-motif due to protonation of the cytidines (Figure 5B), resulting in gel formation (Figure 5C). Addition of base to the system resulted in an immediate gel-to-sol transition. Since the stimulus was just protons in this case, the response of the gel was very quick. One disadvantage of using the i-motif as crosslinker is that the gel is stable only at acidic $\mathrm{pH}$ and therefore cannot be used under physiological conditions. ${ }^{91}$ To address this problem, the authors further engineered the building blocks to contain 8-mer overhangs. Under appropriate stoichiometry, hydrogel formed spontaneously without the need for adding DNA ligase.

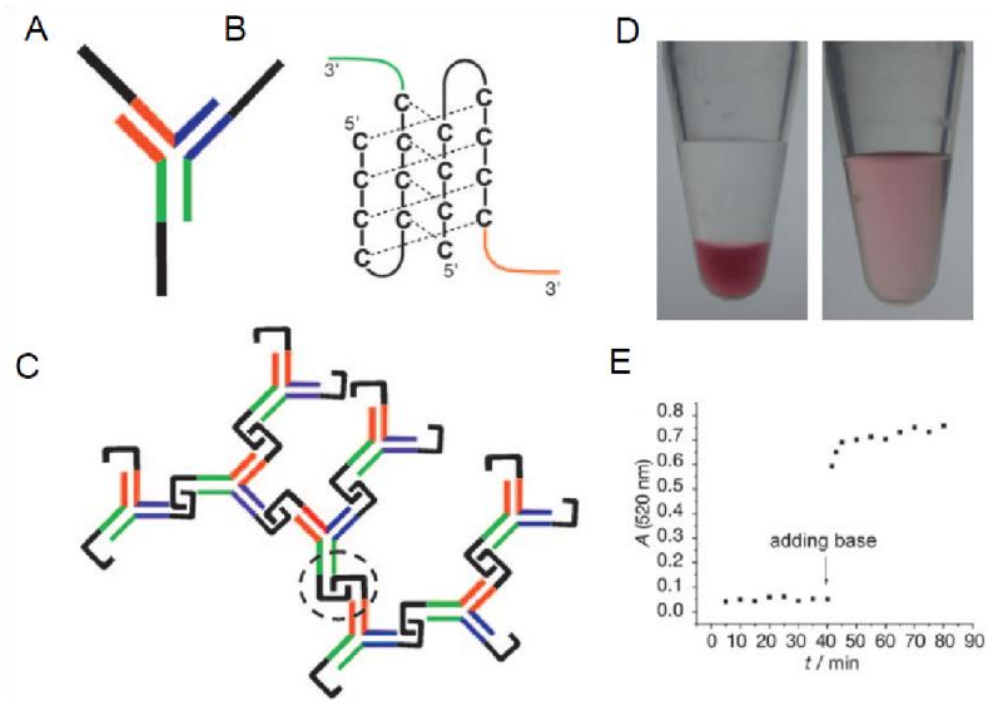

Figure 5. A pH sensitive hydrogel made from pure DNA. (A) A scheme of the three-way junction DNA monomer complex. (B) The formation of i-motif DNA. (C) The linkage of DNA hydrogel. (D) A photograph of hydrogel at low (left) and high $\mathrm{pH}$ (right). (E) Kinetics of color change monitored by UVvis spectrometry upon addition of base to change $\mathrm{pH}$. 


\section{Reversible change of gel property}

While the gel-to-sol transition is easy to observe, such gels cannot be easily regenerated. For example, after addition of a target molecule to dissolve the gel, it is often difficult to remove the molecule to restore the gel state (except for special stimuli such as proton). For many applications it is more desirable to achieve a reversible change of gel property such as volume expansion or shrinkage. Such gels have been demonstrated via protein and antibody attachment. ${ }^{20}$ In the past several years, DNAfunctionalized hydrogels have also been tested using three different strategies. In 2005, Langrana and co-workers reported a system shown in Figure 6A. ${ }^{92}$ A hydrogel was formed with a linker DNA containing a large insertion of nucleotides in the middle (in red). Since the ss-DNA gap was quite flexible, the gel had a low modulus. Addition of the fuel strand (in green) complementary to the gap increased the modulus by forming rigid ds-DNA. Addition of the removal DNA reset the system to its original state. In this whole process, the gel state was maintained since the two ends of the linker DNA responsible for crosslinking the gel were intact.

Murakami and Maeda employed dual modified DNAs with each of the ends labeled with an amino group. ${ }^{93}$ As a result, the gel network was permanently crosslinked unless the DNA chain was cleaved. If the DNA can fold into a hairpin (Figure 6B), the gel was initially in a more compact state due to the short DNA end-to-end distance. Addition of the c-DNA resulted in gel expansion with the formation of rigid ds-DNA. A photograph showing DNA-induced gel volume change is shown in Figure 6D. If the linker DNA did not have any secondary structure (Figure 6C), the authors observed gel shrinkage upon addition of the c-DNA, which was attributed to dehydration associated with the formation of ds-DNA. ${ }^{94}$

As can be observed from Figure 6D, the amount of gel volume change in this process was quite small. In most cases, such volume change was less than $20 \%$, which was comparable to the responsive gels prepared with proteins. Such a small volume change makes it difficult for quantitative visual inspection with the naked eye. To solve this problem, several methods have been tested to improve the 
detection. For example, Tierney and Stokke prepared a hydrogel thin film with a thickness of $\sim 50 \square \mathrm{m}$ on the tip of an optical fiber. Incident light from the fiber was reflected back by the hydrogel and the change of gel volume was converted to the change of the optical path length, which can be sensitively detected by an optical interferometric technique with a resolution of $\sim 2 \mathrm{~nm}$. The hydrogel design is shown in Figure 6E, where the gel was crosslinked with both bis-acrylamide (black dots) and DNA. The DNA linkages can be disrupted by adding a competing strand shown in green. With this technique, even 0.5 $\mu \mathrm{M}$ of the competing strand can be detected. Recently, the effect on the number of DNA base pairs in Figure $6 \mathrm{E}$ has been carefully studied. ${ }^{95}$ As can be expected, the gel swelling kinetics was faster if the competing strand can form more base pairs with the immobilized DNA.

$\mathrm{Gu}$ and co-workers reported an interesting system where a uniform hydrogel film was prepared using silica colloidal crystal beads as a template. ${ }^{96}$ After removal of the beads, the gel had an ordered inverse opal structure. Light reflected by this structure was modulated by gel swelling, which was in turn controlled by the presence of a linker DNA. The gel and DNA design was similar to that shown in Figure 6C. This system showed a remarkable sensitivity of $1 \mathrm{nM}$ target DNA as well as a wide dynamic range up to $1 \mathrm{mM}$ DNA. 
A

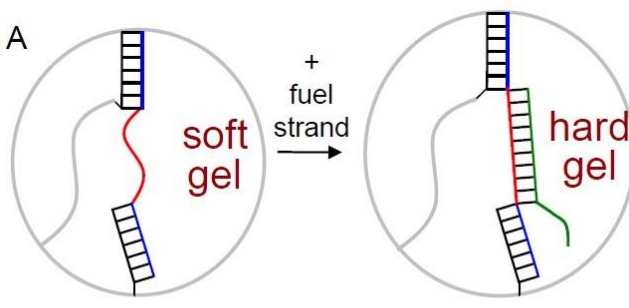

B
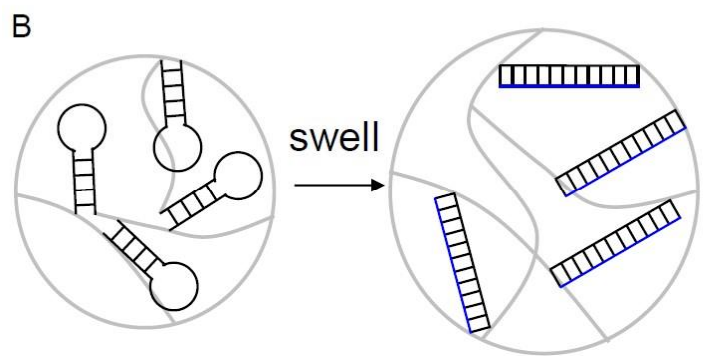

$D$

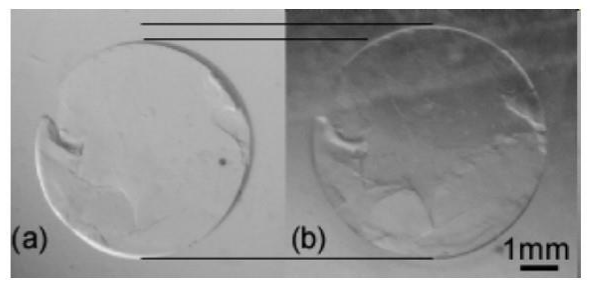

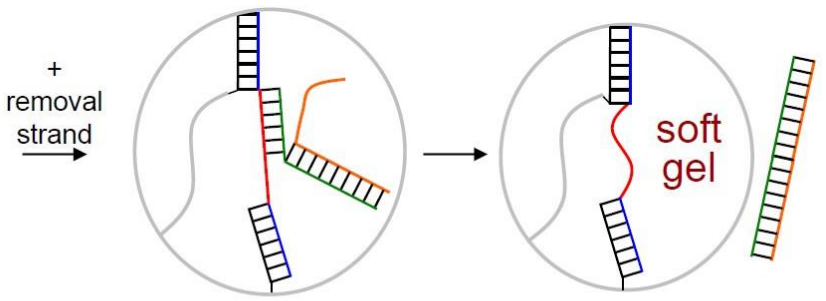

C

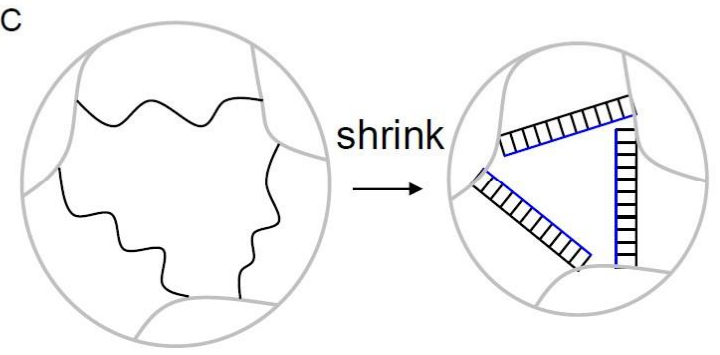

$E$

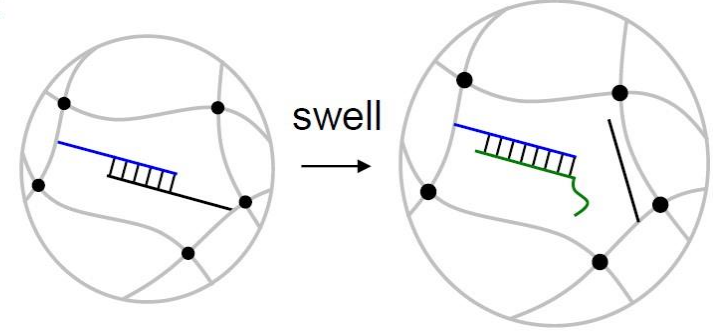

Figure 6. Reversible hydrogel volume change induced by DNA hybridization. (A) By introducing an overhang on the fuel DNA to harden the gel, it is possible to remove the fuel DNA by adding a removal DNA to reversibly control the gel volume. (B) The use of a hairpin DNA to crosslink hydrogel. (C) Addition of the c-DNA induced gel shrinkage where initially the DNA does not have a rigid secondary structure. (D). A photograph of the gel swelling in (B). (E) The gel is crosslinked by both chemical crosslinkers (denoted by black dots) and by DNA. Adding the competing DNA (in green) decreased the number of crosslinks and induced gel swelling.

\section{Hydrogels with optical responses}

In majority of the work reviewed so far, at least $4 \%$ hydrogels were prepared. For polyacrylamide gels, this corresponds to a monomer concentration of $\sim 560 \mathrm{mM}$. To form a gel, the chemical crosslinker (e.g. bis-acrylamide) is typically greater than $0.2 \%$ of the monomer concentration to ensure good mechanical property of the gel. Therefore, if all of the crosslinks have to be established via DNA hybridization, millimolar DNA is needed. Indeed, very high concentrations of DNA were used to prepare most of these 
gels. Even in the case where DNA was used to achieve reversible volume change and crosslinks were established by bis-acrylamide, a similar DNA concentration was required to observe the desired effects. Researchers in the DNA bioanalytical chemistry field routinely work with nM or low $\mu \mathrm{M}$ DNA, which is sufficient to be detected by a fluorometer or by UV-vis spectrophotometer. The thousand to million fold increase in DNA usage is apparently one of the disadvantages of using DNA to prepare such gels.

The need for high DNA concentration appears to be inevitable to prepare traditional stimuli responsive hydrogels using mechanical properties or gel dissolution as an output. On the other hand, a much lower DNA concentration is sufficient for gels with optical outputs. In addition to the cost advantage, the use of a lower concentration of DNA is likely to increase detection sensitivity. My group is interested in designing hydrogels with a visual response and our strategy is to immobilize DNA optical sensors within hydrogels. There are several advantages for using hydrogels for sensor immobilization. First, it has a much larger loading capacity compared to planar surfaces because the immobilization is in 3-D. Second, hydrogels are transparent with a very low optical background, making it easy to observe visual signals. Third, hydrogels can be readily dried, allowing long-term storage. Fourth, the gel matrix can protect the DNA inside the gel from nuclease degradation. Fifth, immobilization also allows for sensor regeneration. Finally, the gel backbone can be engineered to improve sensor performance. All these features have been explored.

We first demonstrated the concept by immobilizing an acrydite-modified $\mathrm{Hg}^{2+}$ binding DNA (see Figure 1D for sequence) in a polyacrylamide hydrogel. ${ }^{97}$ Since the gel was crosslinked by bisacrylamide, only $10 \mu \mathrm{M}$ DNA was used, which was sufficient for visual detection. The signal generation was achieved by adding a DNA intercalation dye SYBR Green I (SG). ${ }^{98}$ We found that in the absence of $\mathrm{Hg}^{2+}$, the gel appeared to be fluorescent yellow while addition of $\mathrm{Hg}^{2+}$ induced green fluorescence (Figure 7A). The immobilized DNA can actively adsorb $\mathrm{Hg}^{2+}$ and thus a sample volumedependent sensitivity was observed. For example, with $1 \mathrm{~mL}$ sample, $200 \mathrm{nM} \mathrm{Hg}^{2+}$ was needed to visually observe while in a 50 
$\mathrm{mL}$ sample, $10 \mathrm{nM} \mathrm{Hg}^{2+}$ can be detected (Figure 7B). The fluorescence intensity can be quantified using a gel documentation system. In this case due to the strong yellow background fluorescence, only 30\% fluorescence increase was observed (Figure 7C). Because of the immobilization, the hydrogel can be easily regenerated using acid treatment and the gel can also be dried for storage and rehydrated prior to use (Figure 7D). The resistance to nuclease degradation was also demonstrated.

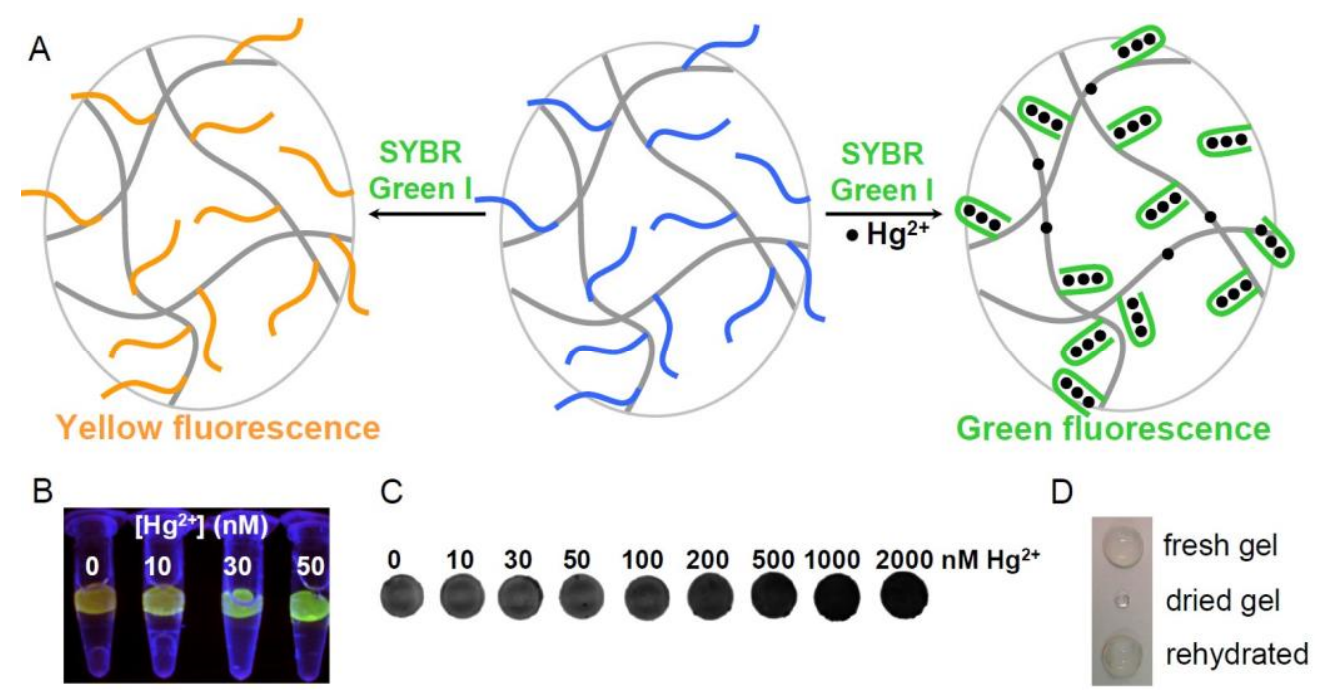

Figure 7. (A) Covalent DNA immobilization in a polyacrylamide hydrogel and its interaction with $\mathrm{Hg}^{2+}$ and SYBR Green I to produce fluorescence signals. (B) The gel sensitivity using $50 \mathrm{~mL}$ samples. (C) Quantification of gel fluorescence intensity using a gel documentation system. (D) Hydrogel drying and rehydration.

Since the DNA was immobilized in a 3-D gel matrix, the property of the gel can influence the interaction between DNA and target molecules. This feature, however, has rarely been explored in the literature. Based on the $\mathrm{Hg}^{2+}$ sensing hydrogel described above, we prepared also cationic gels containing allylamine and anionic gels containing 2-acrylamido-2-methyl-1-propanesulfonic acid (AMPS, see 
Figure $8 \mathrm{~A}$ for structure). ${ }^{99}$ Interestingly, the AMPS gel showed a similar yellow-to-green fluorescence change upon $\mathrm{Hg}^{2+}$ addition, but the cationic gel showed significantly reduced background fluorescence in the absence of $\mathrm{Hg}^{2+}$ (Figure 8B). SG can bind to ds-DNA through strong intercalation and minor groove binding but to ss-DNA through weak electrostatic interactions. In the case of the cationic gel, the SG dye was repelled by the gel and the DNA tended to interact with the gel backbone instead of the dye. Therefore a low fluorescence was achieved. Addition of $\mathrm{Hg}^{2+}$ resulted in ds-DNA formation to which $\mathrm{SG}$ can bind with a high affinity to generate strong green fluorescence. A scheme of this process is shown in Figure 8D. Using the cationic gel containg $20 \%$ allylamine, $~ 300 \%$ fluorescence increase was observed (Figure $8 \mathrm{C})$. The detection limit was determined to be $1.1 \mathrm{nM}$, which was among the highest in all reported $\mathrm{Hg}^{2+}$ sensors.

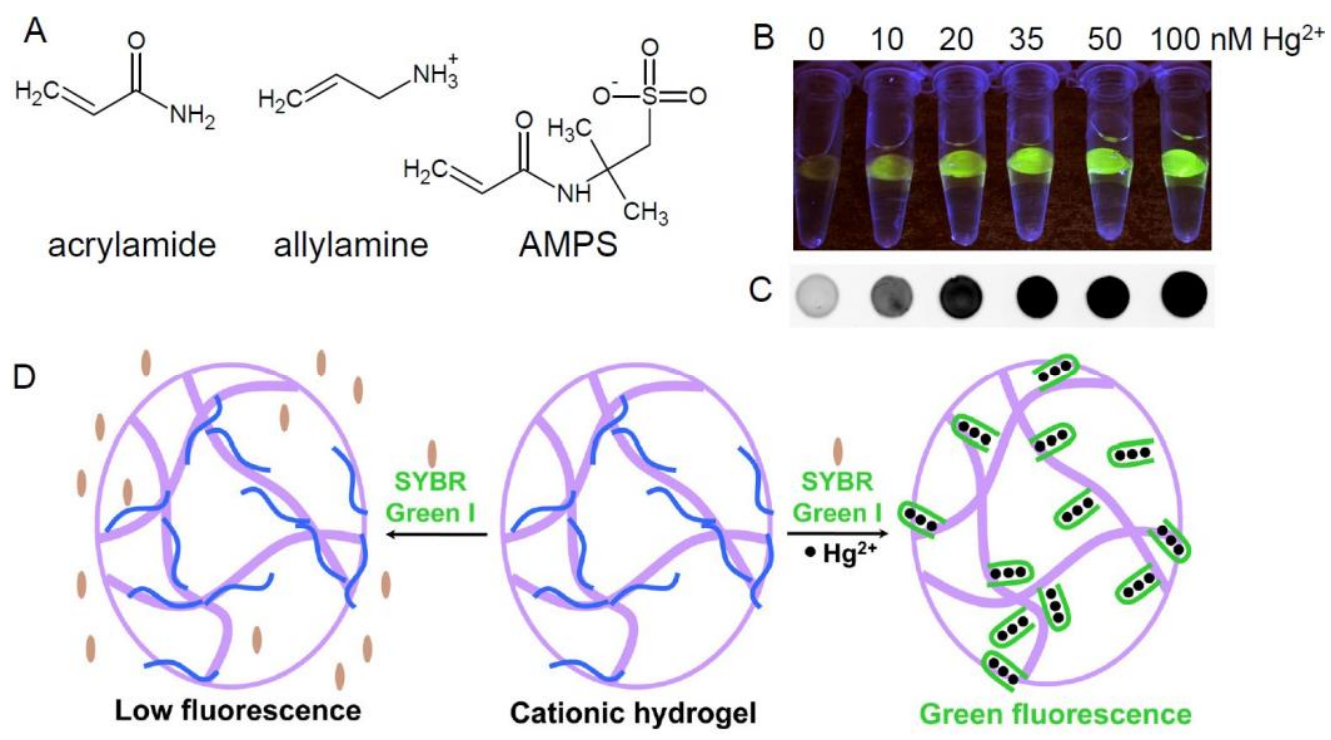

Figure 8. (A) The molecular structures of acrylamide, allylamine, and AMPS. Cationic hydrogel mercury sensor sensitivity obtained using a digital camera (B) and a gel documentation system (C). (D) Schematic presentation of covalent DNA immobilization within a positive hydrogel (containing allylamine). Addition of $\mathrm{Hg}^{2+}$ and $\mathrm{SG}$ produces a visual fluorescence signal. For the cationic gel, the DNA interacts more with the gel backbone in the absence of $\mathrm{Hg}^{2+}$ and the diffusion of SG into the gel is also retarded, giving low background fluorescence. 


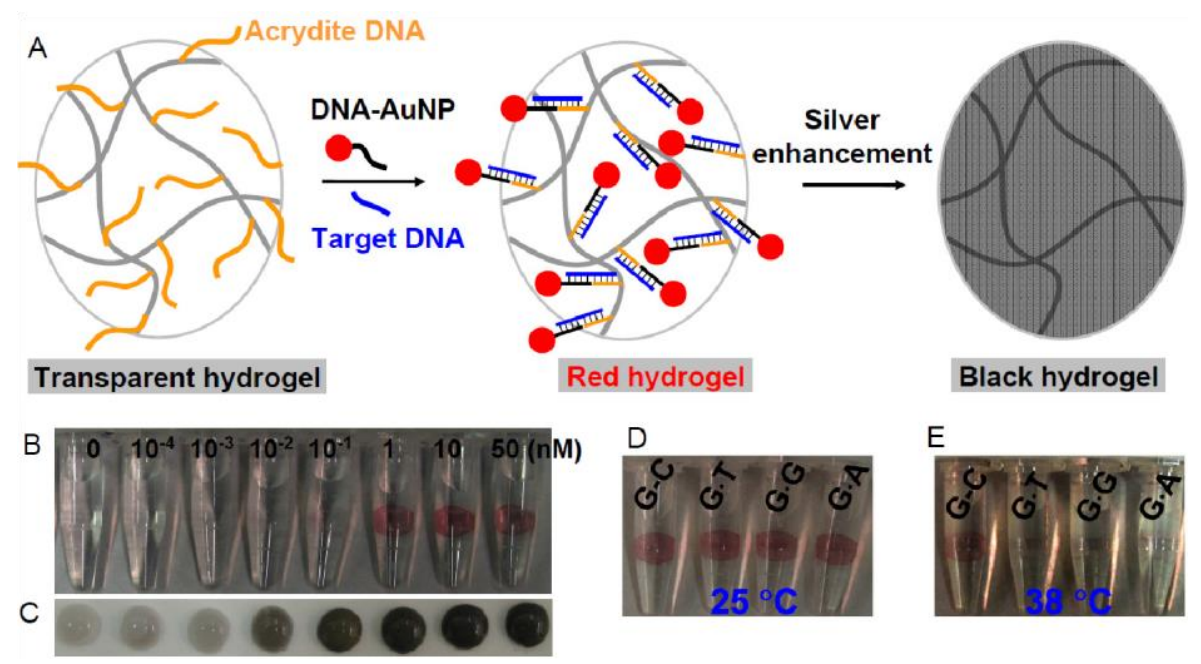

Figure 9. (A) Schematic presentation of using DNA-functionalized hydrogels and AuNPs for colorimetric DNA detection. Transparent gels change color to red in the presence of the target DNA. Signal amplification can be achieved by reducing $\mathrm{Ag}^{+}$in the presence of AuNPs to coat the gel with metallic silver. Sensitivity of DNA detection with AuNPs (B) and after silver enhancement (C) Selectivity test with perfectly matched target DNA and target DNAs containing single base mismatches at $25^{\circ} \mathrm{C}(\mathrm{E})$ and $38^{\circ} \mathrm{C}(\mathrm{F})$.

While visual fluorescence detection is highly sensitive, a UV lamp and a dark room are required. Taking advantage of hydrogels being transparent with a very low optical background and the high extinction coefficients of AuNPs, we also demonstrated colorimetric DNA detection with hydrogels. ${ }^{100}$ In this system, two probe DNAs were used; one immobilized on the hydrogel and the other on AuNPs (Figure 9A). In the presence of the target DNA, the AuNPs were immobilized on the gel giving a red color. With this method, $\sim 1 \mathrm{nM}$ DNA can be visually detected (Figure 9B). The immobilized AuNPs can also be used to catalyze the reduction of $\mathrm{Ag}^{+}$for signal amplification. A detection limit of 1 pM DNA can be achieved with visual inspection (Figure 9C). Since AuNP attachment was realized via 
DNA hybridization, the gels can be regenerated by a simple heat treatment. Furthermore, if the target DNA contained base mismatches, the AuNPs were released at a lower temperature (Figure 9D,E), allowing highly selective DNA detection.
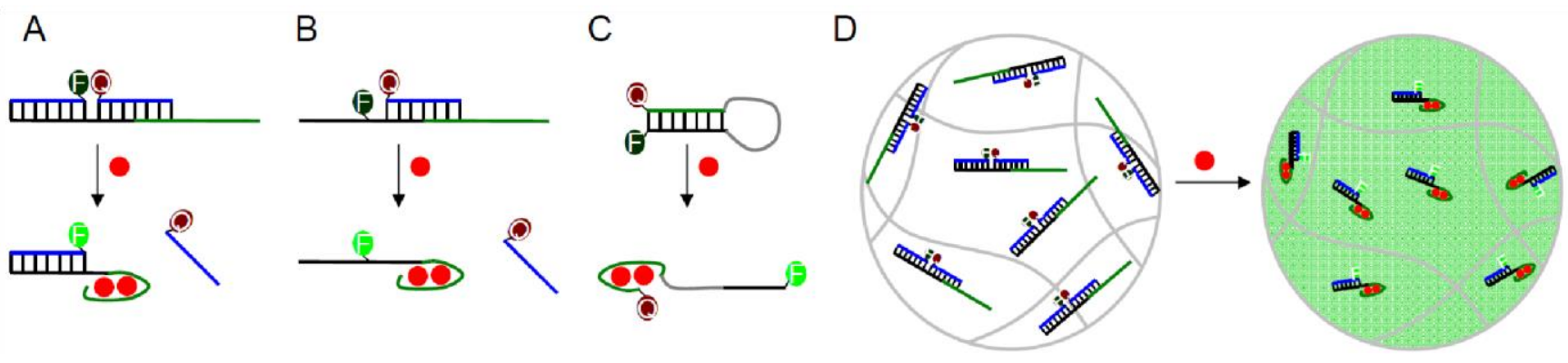

Figure 10. Aptamer based fluorescent sensors for ATP using three DNA strands (A), two DNA strands (B), and one DNA strand (C). Each aptamer can bind two ATP molecules (denoted as a red dot). (D) Schematics of entrapping these DNA sensors in gel and the increase of gel fluorescence in the presence of target molecule.

\section{DNA sensors entrapped in gels.}

In addition to covalent functionalization of hydrogels with DNA, DNA-based sensors have also been physically entrapped in gels. By eliminating the acrydite or amino modifications, the cost of DNA synthesis is reduced. However, such sensors may not be easily regenerated and the entrapped DNA may leach out of the gel matrix. The Li and Brennan groups entrapped fluorescent signaling aptamers in solgel derived silica gel. Two types of sensor designs were tested as shown in Figure 10A and B, where the sensors respectively contained either three or two strands of DNA. ${ }^{101}$ In both cases, the aptamer (in green) binding to its target (ATP) released the quencher-labelled DNA, resulting in fluorescence increase (Figure 8D). The authors demonstrated that DNA leaching can be reduced by the addition of a small fraction of cationic monomer. Interestingly, attaching such signaling aptamers to streptavidin did not improve the retention of the sensor inside gel. Overall a leaching of 12-49\% was observed. Although the 
fluorescence signal increase was slower for the entrapped sensors compared to that for solution phase assays, the final fluorescence increase was similar. The authors further showed the use of such entrapped aptamer sensors to perform high throughput screening for enzyme inhibitors and for metal ion detection. ${ }^{65,102}$

Nielsen et al employed an aptamer sensor design first reported by the Tan group (Figure 8C). ${ }^{103}$ In this case, the sensor is composed of only a single DNA strand which contained a fluorophore, a quencher, the ATP aptamer sequence and a polymer spacer. The sensor was entrapped in $30 \mathrm{~nm}$ diameter polyacryladmide hydrogel nanoparticles, which were delivered into yeast cells to measure the cellular ATP concentration. ${ }^{104}$

\section{Conclusions and future perspectives}

With the invention of solid phase DNA synthesis, PCR, and the availability of a wide range of enzymes that can manipulate DNA, it is now quite easy to prepare various DNA-polymer conjugates with unique structures and functions. ${ }^{105,106}$ Hydrogels are a unique type of polymeric structure with many attractive properties. For example, the volume and phase of hydrogels can be engineered to be sensitive to many environmental conditions including temperature, $\mathrm{pH}$, ionic strength, and solvent composition. On the other hand, DNA is a special polymer; incorporation of DNA into hydrogel can significantly expand the range of stimuli to metal ions, small molecules, nucleic acids, and proteins. The output has also been expanded from the traditional volume change to optical signals. From the examples developed in the past 15 years, the advantages and applications of using DNA as a functional polymer in hydrogel have been clearly demonstrated.

To move the field forward, there are several challenges to be addressed. For example, modified synthetic oligonucleotides are quite expensive if $\mathrm{mM}$ concentrations are required to make hydrogels. One question to be address is to reduce the amount of DNA but can still achieve the desired gel response. Second, hydrogels (especially monoliths) are heterogeneous systems. Many techniques that are routinely 
used for measuring solution fluorescence properties cannot be easily applied to hydrogels. Fluorescence or absorption intensity and wavelength (color) are the major means for detection, which is an advantage in terms of instrument-free detection. The signal change has to be large to be visually detected. Therefore, better sensor designs are needed to accommodate these requirements in a hydrogel. Given the advantages that hydrogels can bring, such studies are well warranted. At the same time, practically useful sensing systems need to be demonstrated to justify the use of DNA to functionalize hydrogels. This will require the isolation of aptamers for biologically and environmentally important targets. Third, most current work employed hydrogel as a matrix for DNA immobilization without paying attention to the effect of gel charge, hydrophobicity, or gel percentage. These studies are not only practically important but also help understand the fundamental interaction between DNA, hydrogel, and small molecules. Finally, there are also technical issues. For example, monolithic hydrogels are bulk and it takes a long time for large chemical stimuli to diffuse in. This has been quite obvious in the examples described above, where protons can induce the gel-to-sol transition in $\sim 1 \mathrm{~min}$, cocaine needs more than $10 \mathrm{~min}$, and thrombin and DNA can take hours. Therefore, the design of gels that can respond quickly to macromolecular input is a challenge. This may be solved by engineering the internal pore structure of hydrogel to make diffusion faster. Alternatively, the size of the gel can be reduced to prepare gel films, ${ }^{107,}{ }^{108}$ as well as hydrogel nanoparticles. ${ }^{104,109,110}$ More advanced applications including using such functionalized gels for tissue engineering and for cell culture can also be envisioned.

Acknowledgment. Funding for the work in Liu lab described in this review is from the University of Waterloo and the Discovery Grant from the Natural Sciences and Engineering Research Council of Canada (NSERC). 


\section{References}

1. R. Langer and D. A. Tirrell, Nature, 2004, 428, 487-492.

2. $\quad$ E. S. Gil and S. M. Hudson, Progress in Polymer Science, 2004, 29, 1173-1222.

3. Y. Qiu and K. Park, Adv. Drug Deliver. Rev., 2001, 53, 321-339.

4. $\quad$ K. Y. Lee and D. J. Mooney, Chem. Rev., 2001, 101, 1869-1879.

5. $\quad$ B. Jeong, S. W. Kim and Y. H. Bae, Adv. Drug Deliver. Rev., 2002, 54, 37-51.

6. A. S. Hoffman, Adv. Drug Deliver. Rev., 2002, 54, 3-12.

7. W. E. Hennink and C. F. van Nostrum, Adv. Drug Deliver. Rev., 2002, 54, 13-36.

8. C. Tsitsilianis, Soft Matter, 2010, 6, 2372-2388.

9. S. Banta, I. R. Wheeldon and M. Blenner, Annu. Rev. Biomed. Eng., 2010, 12, 167-186.

10. Y. Osada, H. Okuzaki and H. Hori, Nature, 1992, 355, 242-244.

11. S. Nayak and L. A. Lyon, Angew. Chem. Int. Ed., 2005, 44, 7686-7708.

12. X. Wang, X. Qiu and C. Wu, Macromolecules, 1998, 31, 2972-2976.

13. N. A. Peppas, J. Z. Hilt, A. Khademhosseini and R. Langer, Adv. Mater., 2006, 18, 1345-1360.

14. N. A. Peppas, P. Bures, W. Leobandung and H. Ichikawa, Eur. J. Pharm. Biopharm., 2000, 50, 27-46.

15. K. Gawel, D. Barriet, M. Sletmoen and B. T. Stokke, Sensors, 2010, 10, 4381-4409.

16. G. R. Hendrickson, M. H. Smith, A. B. South and L. A. Lyon, Adv. Funct. Mater., 2010, 20, 1697-1712.

17. D. T. Eddington and D. J. Beebe, Adv. Drug Deliver. Rev., 2004, 56, 199-210.

18. T. Miyata, N. Asami and T. Uragami, Nature, 1999, 399, 766-769.

19. C. Wang, R. J. Stewart and J. Kopecek, Nature, 1999, 397, 417-420. 
20. W. L. Murphy, W. S. Dillmore, J. Modica and M. Mrksich, Angew. Chem. Int. Ed., 2007, 46, 3066-3069.

21. M. Ehrbar, R. Schoenmakers, E. H. Christen, M. Fussenegger and W. Weber, Nat. Mater., 2008, 7, 800-804.

22. N. C. Seeman, Nature, 2003, 421, 427-431.

23. C. Lin, Y. Liu and H. Yan, Biochemistry, 2009, 48, 1663-1674.

24. F. A. Aldaye, A. L. Palmer and H. F. Sleiman, Science, 2008, 321, 1795-1799.

25. A. P. Alivisatos, K. P. Johnsson, X. Peng, T. E. Wilson, C. J. Loweth, M. P. Bruchez, Jr and P. G. Schultz, Nature, 1996, 382, 609-611.

26. C. A. Mirkin, R. L. Letsinger, R. C. Mucic and J. J. Storhoff, Nature, 1996, 382, 607-609.

27. J. J. Storhoff and C. A. Mirkin, Chem. Rev., 1999, 99, 1849-1862.

28. J. Liu, Z. Cao and Y. Lu, Chem. Rev., 2009, 109, 1948-1998.

29. P. W. K. Rothemund, Nature, 2006, 440, 297.

30. J. P. Zheng, J. J. Birktoft, Y. Chen, T. Wang, R. J. Sha, P. E. Constantinou, S. L. Ginell, C. D. Mao and N. C. Seeman, Nature, 2009, 461, 74-77.

31. S. Y. Park, A. K. R. Lytton-Jean, B. Lee, S. Weigand, G. C. Schatz and C. A. Mirkin, Nature, 2008, 451, 553-556.

32. D. Nykypanchuk, M. M. Maye, D. van der Lelie and O. Gang, Nature, 2008, 451, 549-552.

33. J. Sharma, R. Chhabra, A. Cheng, J. Brownell, Y. Liu and H. Yan, Science, 2009, 323, 112-116.

34. C. Tuerk and L. Gold, Science, 1990, 249, 505-510.

35. A. D. Ellington and J. W. Szostak, Nature, 1990, 346, 818-822.

36. D. L. Robertson and G. F. Joyfce, Nature, 1990, 344, 467-468.

37. U. Feldkamp and C. M. Niemeyer, Angew. Chem., Int. Ed., 2006, 45, 1856-1876.

38. E. Katz and I. Willner, Angew. Chem., Int. Ed., 2004, 43, 6042-6108. 
39. Y. Lu and J. Liu, Curr. Opin. Biotechnol., 2006, 17, 580-588.

40. Y. Lu and J. Liu, Acc. Chem. Res., 2007, 40, 315-323.

41. S. Nagahara and T. Matsuda, Polymer Gels and Networks, 1996, 4, 111-127.

42. D. C. Lin, B. Yurke and N. A. Langrana, in [Engineering in Medicine and Biology, 2002. 24th Annual Conference and the Annual Fall Meeting of the Biomedical Engineering Society] EMBS/BMES Conference, 2002. Proceedings of the Second Joint, 2002, pp. 627-628 vol.621.

43. M. C. Moran, F. R. Baptista, A. Ramalho, M. G. Miguel and B. Lindman, Soft Matter, 2009, 5, 2538-2542.

44. M. C. Moran, M. G. Miguel and B. Lindman, Langmuir, 2007, 23, 6478-6481.

45. M. C. Moran, M. G. Miguel and B. Lindman, Biomacromolecules, 2007, 8, 3886-3892.

46. M. C. Moran, M. G. Miguel and B. Lindman, Soft Matter, 2010, 6, 3143-3156.

47. M. C. Moran, M. R. Infante, M. G. Miguel, B. Lindman and R. Pons, Langmuir, 2010, 26, 10606-10613.

48. D. Costa, A. J. M. Valente, A. Pais, M. G. Miguel and B. Lindman, Colloid. Surface. A., 2010, 354, 28-33.

49. F. Topuz and O. Okay, Biomacromolecules, 2009, 10, 2652-2661.

50. $\quad$ N. Orakdogen, B. Erman and O. Okay, Macromolecules, 2010, 43, 1530-1538.

51. H. W. Tang, X. R. Duan, X. L. Feng, L. B. Liu, S. Wang, Y. L. Li and D. B. Zhu, Chem. Comm., 2009, 641-643.

52. T. Amiya and T. Tanaka, Macromolecules, 1987, 20, 1162-1164.

53. F. Horkay and P. J. Basser, Biomacromolecules, 2003, 5, 232-237.

54. Y. Xu, Q. Wu, Y. Sun, H. Bai and G. Shi, Acs Nano, 2010, null-null.

55. S. H. Nezhadi, P. F. M. Choong, F. Lotfipour and C. R. Dass, Journal of Drug Targeting, 2009, 17, $731-738$. 
56. H. Hall, Current Pharmaceutical Design, 2007, 13, 3597-3607.

57. M. Goldberg, R. Langer and X. Q. Jia, Journal of Biomaterials Science-Polymer Edition, 2007, 18, 241-268.

58. D. E. Huizenga and J. W. Szostak, Biochemistry, 1995, 34, 656-665.

59. M. N. Stojanovic, P. de Prada and D. W. Landry, J. Am. Chem. Soc., 2001, 123, 4928-4931.

60. Y. Miyake, H. Togashi, M. Tashiro, H. Yamaguchi, S. Oda, M. Kudo, Y. Tanaka, Y. Kondo, R. Sawa, T. Fujimoto, T. Machinami and A. Ono, J. Am. Chem. Soc., 2006, 128, 2172.

61. A. Ono and H. Togashi, Angew. Chem., Int. Ed., 2004, 43, 4300-4302.

62. D. S. Wilson and J. W. Szostak, Annu. Rev. Biochem., 1999, 68, 611-647.

63. S. D. Jayasena, Clin. Chem, 1999, 45, 1628-1650.

64. F. N. Rehman, M. Audeh, E. S. Abrams, P. W. Hammond, M. Kenney and T. C. Boles, Nucleic Acids Res., 1999, 27, 649-655.

65. Y. Shen, G. Mackey, N. Rupcich, D. Gloster, W. Chiuman, Y. Li and J. D. Brennan, Anal. Chem., 2007, 79, 3494-3503.

66. B. Soontornworajit, J. Zhou and Y. Wang, Soft Matter, 2010, 6, 4255-4261.

67. R. Elghanian, J. J. Storhoff, R. C. Mucic, R. L. Letsinger and C. A. Mirkin, Science, 1997, 277, 1078-1080.

68. J. J. Storhoff, R. Elghanian, R. C. Mucic, C. A. Mirkin and R. L. Letsinger, J. Am. Chem. Soc., 1998, 120, 1959-1964.

69. N. L. Rosi and C. A. Mirkin, Chem. Rev., 2005, 105, 1547-1562.

70. D. C. Lin, B. Yurke and N. A. Langrana, Journal of Biomechanical Engineering, 2004, 126, 104-110.

71. B. Yurke, D. C. Lin and N. A. Langrana, in DNA Computing, eds. A. Carbone and N. A. Pierce, 2006, pp. 417-426. 
72. T. Liedl, H. Dietz, B. Yurke and F. Simmel, Small, 2007, 3, 1688-1693.

73. J. W. Liu and Y. Lu, Angew. Chem. Int. Ed., 2006, 45, 90-94.

74. C.-C. Huang, Y.-F. Huang, Z. Cao, W. Tan and H.-T. Chang, Anal. Chem., 2005, 77, 57355741.

75. V. Pavlov, Y. Xiao, B. Shlyahovsky and I. Willner, J. Am. Chem. Soc., 2004, 126, 11768-11769.

76. J. Liu and Y. Lu, Adv. Mater., 2006, 18, 1667-1671.

77. J. W. Liu, J. H. Lee and Y. Lu, Anal. Chem., 2007, 79, 4120-4125.

78. M. V. Yigit, D. Mazumdar, H.-K. Kim, J. H. Lee, B. Odintsov and Y. Lu, ChemBioChem, 2007, 8, 1675-1678.

79. H. H. Yang, H. P. Liu, H. Z. Kang and W. H. Tan, J. Am. Chem. Soc., 2008, 130, 6320-6321.

80. Z. Zhu, C. C. Wu, H. P. Liu, Y. Zou, X. L. Zhang, H. Z. Kang, C. J. Yang and W. H. Tan, Angew. Chem. Int. Ed., 2010, 49, 1052-1056.

81. H. Kang, H. Liu, X. Zhang, J. Yan, Z. Zhu, L. Peng, H. Yang, Y. Kim and W. Tan, Langmuir, 2011, 27, 399-408.

82. H. Asanuma, X. Liang, H. Nishioka, D. Matsunaga, M. Liu and M. Komiyama, Nat. Protocols, 2007, 2, 203-212.

83. B. Wei, I. Cheng, K. Q. Luo and Y. Mi, Angew. Chem., Int. Ed., 2008, 47, 331-333.

84. X. He, B. Wei and Y. Mi, Chem. Comm., 2010, 46, 6308-6310.

85. B. Soontornworajit, J. Zhou, M. T. Shaw, T. H. Fan and Y. Wang, Chem. Comm., 2010, 46, 1857-1859.

86. S. Venkatesh, J. Wower and M. E. Byrne, Bioconjug. Chem., 2009, 20, 1773-1782.

87. S. Keller, J. Wang, M. Chandra, R. d. Berger and A. Marx, J. Am. Chem. Soc., 2008, 130, 13188-13189.

88. S. H. Um, J. B. Lee, N. Park, S. Y. Kwon, C. C. Umbach and D. Luo, Nat. Mater., 2006, 5, 
797-801.

89. $\quad$ N. Park, S. H. Um, H. Funabashi, J. Xu and D. Luo, Nat. Mater., 2009, 8, 432-437.

90. E. J. Cheng, Y. Z. Xing, P. Chen, Y. Yang, Y. W. Sun, D. J. Zhou, L. J. Xu, Q. H. Fan and D. S. Liu, Angew. Chem. Int. Ed., 2009, 48, 7660-7663.

91. Y. Xing, E. Cheng, Y. Yang, P. Chen, T. Zhang, Y. Sun, Z. Yang and D. Liu, Adv. Mater., 2011, in press, doi: 10.1002/adma.201003343.

92. D. C. Lin, B. Yurke and N. A. Langrana, J. Mater. Res., 2005, 20, 1456-1464.

93. Y. Murakami and M. Maeda, Biomacromolecules, 2005, 6, 2927-2929.

94. Y. Murakami and M. Maeda, Macromolecules, 2005, 38, 1535-1537.

95. M. Gao, K. Gawel and B. T. Stokke, Soft Matter, 2011, in press, doi: 10.1039/C0SM00915F.

96. Y. J. Zhao, X. W. Zhao, B. C. Tang, W. Y. Xu, J. Li, L. Hu and Z. Z. Gu, Adv. Funct. Mater., 2010, 20, 976-982.

97. N. Dave, P.-J. J. Huang, M. Y. Chan, B. D. Smith and J. Liu, J. Am. Chem. Soc., 2010, 132, $12668-12673$.

98. J. Wang and B. Liu, Chem. Comm., 2008, 4759-4761.

99. K. A. Jesoph, N. Dave and J. Liu, ACS Appl. Mater. Inter., 2011, in press, doi: 10.1021/am101068c.

100. A. Baeissa, N. Dave, B. D. Smith and J. Liu, ACS Appl. Mater. Inter., 2010, 2, 3594-3600.

101. N. Rupcich, R. Nutiu, Y. Li and J. D. Brennan, Anal. Chem., 2005, 77, 4300-4307.

102. N. Rupcich, R. Nutiu, Y. Li and J. D. Brennan, Angew. Chem., Int. Ed., 2006, 45, 3295-3299.

103. Z. W. Tang, P. Mallikaratchy, R. H. Yang, Y. M. Kim, Z. Zhu, H. Wang and W. H. Tan, J. Am. Chem. Soc., 2008, 130, 11268-11269.

104. L. J. Nielsen, L. F. Olsen and V. C. Ozalp, Acs Nano, 2010, 4, 4361-4370.

105. F. E. Alemdaroglu and A. Herrmann, Org. Biomol. Chem., 2007, 5, 1311-1320. 
106. M. Kwak and A. Herrmann, Angew. Chem. Int. Ed., 2010, 49, 8574-8587.

107. I. Tokarev and S. Minko, Soft Matter, 2009, 5, 511-524.

108. C. Carrasquilla, Y. Li and J. D. Brennan, Anal. Chem., 2011, 83, 957-965.

109. D. Gao, H. Xu, M. A. Philbert and R. Kopelman, Angew. Chem. Int. Ed., 2007, 46, 2224-2227.

110. J. Kim, C.-A. Im, Y. Jung, A. Qazi and J.-S. Shin, Biomacromolecules, 2010, 11, 1705-1709. 